\title{
A Mobile Starfield Visualization with Space Compression Capabilities
}

\author{
Stefano Burigat \\ stefano.burigat@dimi.uniud.it
}

\author{
Luca Chittaro \\ luca.chittaro@dimi.uniud.it
}

\author{
$\mathrm{HCl} \mathrm{Lab}$ \\ Department of Mathematics and Computer Science \\ University of Udine \\ Via delle Scienze 206, 33100 \\ Udine, Italy
}

\begin{abstract}
In this paper, we present MoStarD, a mobile application that couples starfield displays with dynamic queries to support user exploration of large datasets. A novel space compression technique enables users to compress areas of the starfield display to optimize screen space usage and easily focus on specific subsets of a dataset.
\end{abstract}

\section{Categories and Subject Descriptors}

H.3.3 [Information Search and Retrieval]: Information filtering, query formulation; H.5.2 [User Interfaces]: Graphical user interfaces (GUI), interaction styles, prototyping, screen design

\section{General Terms}

Design, Human Factors

\section{Keywords}

Information visualization, starfield displays, small-screen devices

\section{INTRODUCTION}

Starfield displays combine 2D scatterplots for visual information presentation with additional features to support selection and zooming [1]. When coupled with dynamic queries, starfield displays are a powerful tool to explore datasets of multi-attribute specify queries and visually analyze results.

Despite the potential benefits, very few attempts have been made at adapting starfield displays and dynamic queries to the mobile domain. One of the first mobile implementations of these techniques [4] visualized a small movie database on a palmtop and allowed users to filter movies by genre and obtain details by tapping single movie elements. In [3], this approach has been enhanced by providing comprehensive panning and zooming capabilities as well as enabling users to change the variable associated to each axis. A subsequent work [2] compared two alternative interaction techniques to prune visual clutter in a starfield display and to make each item easily accessible to the user.

In this paper, we describe MoStarD (Mobile Starfield Display), a flexible mobile visualization and data exploration solution based on starfield displays and dynamic queries that integrates a novel space compression technique to optimize screen space usage. elements, providing users with a fast and easy-to-use method to

\section{INTERACTING WITH MOSTARD}

In MoStarD, users first choose a mapping between attributes of dataset elements and properties of corresponding starfield elements by operating simple dropdown lists. Then, they explore displayed data by manipulating query devices, selecting individual elements or compressing space.

Figure 1 illustrates MoStarD main screen after the mapping step. Two main components are clearly recognizable: a scatterplot and two dynamic query widgets. The scatterplot takes most of the space, and initially shows all the elements in the selected dataset (Fig. 1a), while the two widgets, close to the $x$ and $y$ axes, allow users to filter displayed elements. MoStarD supports two types of query widget, the rangeslider, which is associated to numeric attributes and allows one to change the range of values of the related attribute by acting on two independent handles, and the alphaslider, which allows one to rapidly scan through and select from lists of alphanumeric data. By manipulating sliders, users specify constraints on the corresponding attributes and the scatterplot displays a zoomed view of those elements that satisfy the query. For example, the elements displayed in Fig. 1b are the subset of the whole dataset displayed in Fig. 1a that satisfies the current query (i.e., elements whose value falls in the $\{27-31\}$ range for the "Age" attribute and in the $\{28-37\}$ range for the "Points" attribute). This automatic zoom makes it easier for users to compare and select elements having the specified properties

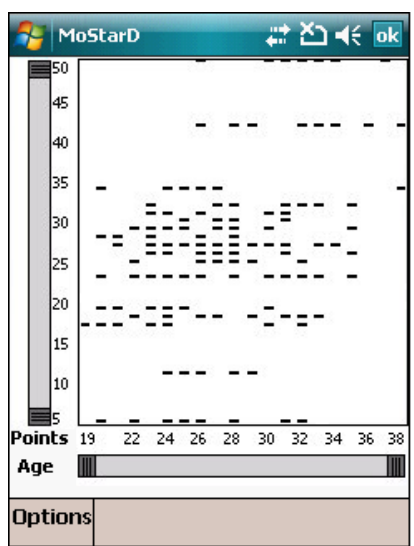

(a)

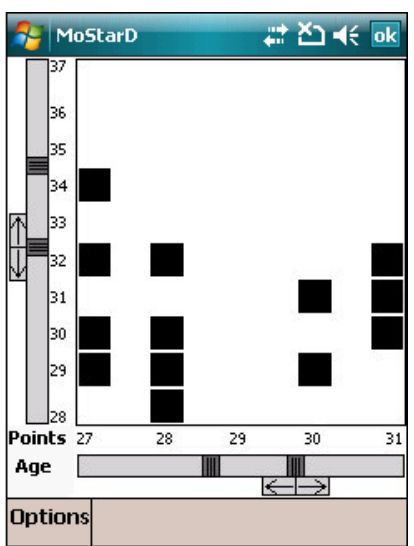

(b)
Figure 1: When users manipulate query devices, MoStarD zooms on those elements that satisfy the query. 


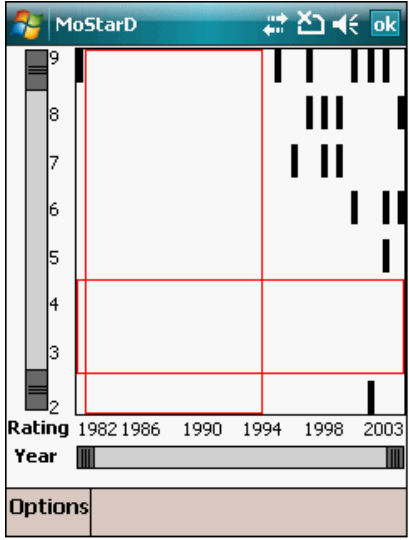

(a)

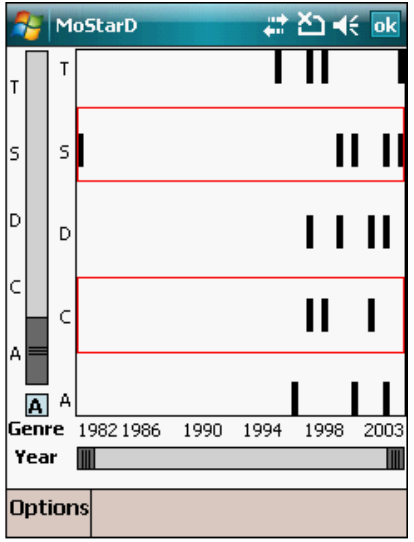

(b)
Figure 2: Examples of possible selections: combination of horizontal and vertical regions (a), disjoint regions (b).

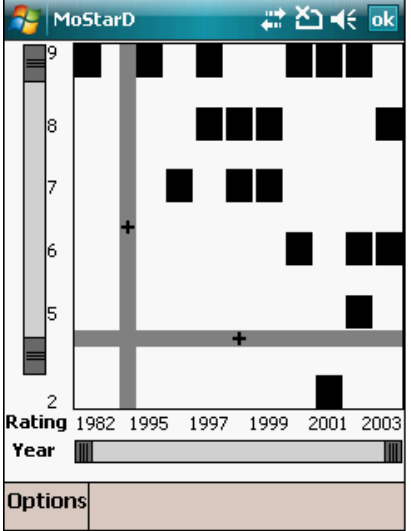

(a)

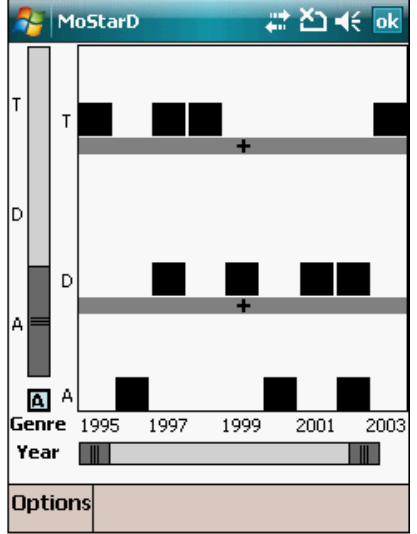

(b)
Figure 3: Result of space compression for the examples in Fig. 2.

because their size typically increases with respect to the initial view. When users tap on an element on the scatterplot, they obtain a screen providing details on that element.

To improve user flexibility in exploring a dataset on mobile devices, we introduce a novel technique based on space compression. Exploiting space compression involves a two-steps process. In the first step, users highlight the regions they want to compress in the scatterplot. To highlight a region, they tap on its starting point and drag the pen towards its ending point. When the desired width is reached, users lift the pen and MoStarD highlights the resulting region by means of a red outline (Fig. 2). Users can easily highlight a combination of horizontal and vertical regions (Fig. 2a) as well as disjoint regions (Fig. 2b).

In the second step, users activate space compression by simply tapping on any point within a region or by selecting a menu item in the main menu. The system replaces each highlighted region with a rectangular placeholder, as illustrated in Fig. 3. At any time, users can incrementally add new regions to compress, or decompress compressed regions by tapping on placeholders.

Figures 2 and 3 illustrate two different ways users can take advantage of MoStarD space compression capabilities. Figure 2a shows a movie dataset with movie rating mapped to the y axis and release year mapped to the $\mathrm{x}$ axis. In this case, space compression allows the user to hide empty regions, obtaining the result displayed in Fig. 3a. Elements on the scatterplot are reorganized to take advantage of the space freed by hidden elements: this simplifies element comparison and selection and is similar to a sort of multi-point zoom.

The example in Fig. 2b shows the same movie dataset, with movie genre mapped to the $y$ axis and release year mapped to the $x$ axis. In this case, the user compresses two populated regions (those corresponding to science fiction and comedy movies) because she wants to focus on thriller, drama, and action movies. Beside optimizing screen space usage, the result of this compression, displayed in Fig. 3b, excludes those movies that are not part of the current user focus, thus reducing user's analysis effort.

It should be noted that current starfield displays do not provide users with the capability to zoom on disjoint sets of elements as in MoStarD.

\section{CONCLUSIONS AND FUTURE WORK}

This paper showed how it is possible to couple starfield displays with dynamic queries on mobile devices. The proposed approach allows users to look at the effect of queries in real-time while manipulating query devices. The novel space compression technique we introduced increases user flexibility in exploring a dataset and further optimizes screen space usage. MoStarD usability has been informally checked on a few users with positive results. We now plan to carry out a comprehensive evaluation, also comparing MoStarD with the starfield displays for mobile devices described in Section 1.

\section{ACKNOWLEDGMENTS}

We acknowledge the financial support of the Italian Ministry of Education, University and Research (MIUR) within the FIRB project number RBIN04M8S8.

\section{REFERENCES}

[1] C. Ahlberg and B. Shneiderman. Visual information seeking: Tight coupling of dynamic query filters with starfield displays. In Proc. Conference on Human Factors in Computing Systems (CHI 94), pages 313-317. ACM Press, 1994.

[2] T. Buring, J. Gerken, and H. Reiterer. User Interaction with Scatterplots on Small Screens - A Comparative Evaluation of Geometric-Semantic Zoom and Fisheye Distortion. IEEE Transactions on Visualization and Computer Graphics, 12(5):829-836, 2006.

[3] T. Buring and $\mathrm{H}$. Reiterer. Zuiscat: querying and visualizing information spaces on personal digital assistants. In Proc. Conference on Human-Computer Interaction with Mobile Devices and Services (MobileHCI 2005), pages 129-136. ACM Press, 2005.

[4] M.D. Dunlop and N. Davidson. Visual information seeking on palmtop devices. In Proc. Conference of the British HCI Group (HCI 2000), pages 19-20. Springer-Verlag, 2000. 